\title{
Solid-fluid interaction in path instabilities of sedimenting flat objects
}

\author{
Jan Dušek, Wei Zhou, and Marcin Chrust \\ Université de Strasbourg, Institut ICube - Equipe de Mécanique des Fluides, 2, rue \\ Boussingault, F-67000 Strasbourg, France \\ dusek@unistra.fr
}

\begin{abstract}
As soon as particles, freely falling or ascending under the action of gravity, buoyancy and hydrodynamic forces, reach Reynolds numbers exceeding values of the order of one hundred, path instabilities arise yielding non vertical, mostly oscillating, trajectories. In this paper we focus on the behavior of flat undeformable solid spheroids. Direct numerical simulations of their fall (ascension) in a quiescent ambient Newtonian fluid reveal the existence of regimes characterized by strong and weak interaction between the fluid and the solid body motion resulting, respectively, in strongly oscillating or almost straight trajectories. In many cases these regimes even co-exist for the same problem parameters. Mathematically, this makes their prediction dependent on initial conditions and, physically, this may result in a strong sensitivity of solid-fluid systems to external perturbations.
\end{abstract}

Keywords: solid-fluid interaction, path instabilities, oblate spheroids

\section{Introduction}

Path instabilities considerably change the behavior both of individual sedimenting particles and of a whole multi-particle system. At the scale of a single particle, this leads to a multitude of non-trivial regimes. These effects are especially striking for particles falling vertically in laminar regime (spheres, flat axisymmetric bodies, oblate spheroids, cylinders, etc.). At large scales of sedimenting systems of a large number of particles, the path instabilities have been recognized to cause clustering in dilute systems leading to otherwise unpredicted increase of sedimenting velocities [1]. Both, the single particle and multi-particle systems depend on the intensity of the effective coupling between the wake and the degrees of freedom of the solid body. The latter results in regimes with strong and weak response of the body dynamics to that of the fluid flow.

The earliest relatively exhaustive parametric study of path instabilities dates back to early 2000s [2] and concerned homogenous spherical bodies (considered also in ref. [1]). However, homogeneous spheres appear to be too perfect to present as rich dynamics as flat objects [3]. Strongly and weakly oscillating trajectories were found in direct numerical simulations of infinitely flat disks [4] and flat cylinders of large diameter/height aspect ratios [5]. At the same time, the 
strongly and weakly interacting modes were predicted for flat cylinders by linear analysis by Tchoufag et al. [6] who introduced the terminology of, respectively, 'solid' and 'fluid' modes.

A detailed parametric study was presented recently for oblate spheroids of aspect ratio $\chi=d / a$, where $d$ is the equatorial diameter and $a$ the length of the symmetry axis, varying from infinity to one [7]. It benefits from a specifically designed numerical method [4] based on spectral - spectral-element spatial discretization combined with a domain decomposition using a dynamic sub-domain reconnection based on the spherical harmonic expansion yielding a highly accurate and highly efficient solver. Among other results, the investigation revealed many cases of co-existence of solid and fluid modes. This co-existence considerably influences the transition scenario. The latter is parameterized by three parameters obtained by non-dimensionalizing the flow equations coupled with solid body motion equations. In addition to the aspect ratio characterizing the shape of spheroids (assumed to be homogeneous), two more parameters were defined in ref. [7]. The relative inertia of the body and of the surrounding fluid is determined by the non-dimensionalized mass $m^{*}=m /\left(\rho d^{3}\right)$ where $m$ is the mass of the spheroid, $d$ its diameter and $\rho$ the fluid density. The third parameter is the Reynolds number $G=U d / \nu$ based on the velocity scale $U$ given by the effective acceleration due to gravity and buoyancy $U=\sqrt{\left(m^{*}-V / d^{3}\right) g d}$ ( $\nu$ being the kinematic viscosity of the fluid and $V$ the volume of the body) called Galileo number. In the investigated parameter domain, the average vertical asymptotic velocities non-dimensionalized by this velocity scale lie between one and two which means that the asymptotic Reynolds numbers can be roughly estimated to be situated between once and twice the Galileo number. The threeparameter domain investigated in ref. [7] is delimited by $\chi \geq 1.1$ (almost spheres to infinitely flat spheroids), $m^{*} \leq 5$ (at $m^{*}=5$, the behavior is expected to be qualitatively similar to any larger $m^{*}$ ) and $30 \leq G \leq 300$. The lowest instability threshold was found for the infinitely flat spheroid of $m^{*}=2$ at $G=35$. The investigation was limited to $G \leq 300$. As a consequence, not all regimes become chaotic, however, most thresholds delimiting individual regimes become almost independent of the Galileo number at this limit.

The mentioned paper [7] evidences significant sub-critical effects resulting in many cases of co-existence of multiple stable states. Mostly, the coexisting states are characterized by strong and weak solid-fluid interaction. No place was available to discuss and illustrate their differences. In the present paper we select three qualitatively very different examples of co-existing fluid and solid states and show the impact of the different solid - fluid interactions on the wake and trajectory of the falling body.

\section{Some examples of co-existing states with strong and weak solid-fluid interaction}

Three examples for three different aspect ratios of spheroids are considered in what follows. The first one concerns very flat spheroids of aspect ratio 10 of 
$m^{*}=0.25$ at Galileo numbers 115 (see figure 1). Note that, unlike in figure 2 of ref. [7], both regimes are situated exactly at the same point of the parameter space. The critical Galileo number for the loss of stability of the steady vertical trajectory is 91 for the same aspect ratio independently of of $m^{*}$ for $m^{*}<0.3$. 'Light' spheroids obey the sphere-like scenario with a regular (steady) primary bifurcation. As can be seen, the fluid mode is characterized by a very small amplitude of oscillation of the spheroid axis as compared to the solid mode. In agreement with the sphere-like scenario, the trajectory is actually oblique oscillating, albeit with a very slight mean inclination of only 0.3 degrees with respect to the vertical. In contrast, the extent of vorticity iso-surfaces taken at the same level of \pm 0.7 is comparable and the maximum (situated between 0.5 and $2 d$ downstream of the body center) is of the same order, respectively, 2.7 and 6.9 , for the fluid and the solid mode. In the fluid mode, the wake structures as practically the same as that of a fixed body with significant streamwise vorticity due to the vortex shedding but with negligible response of the body. In the solid mode, the response of the body to the wake oscillation is very strong. It further enhances the wake vorticity and, consequently, the drag, leading to a reduction of mean settling velocity by $14 \%$.

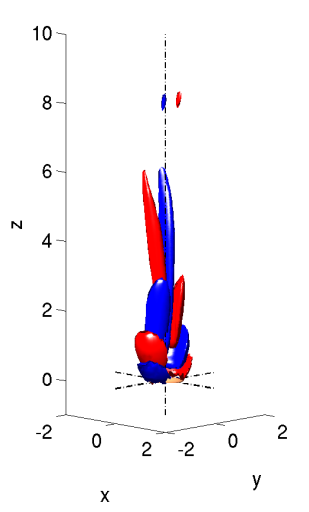

a)

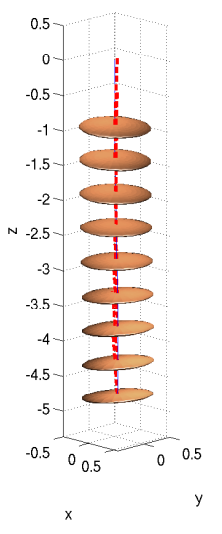

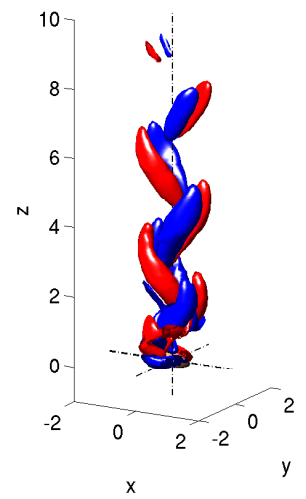

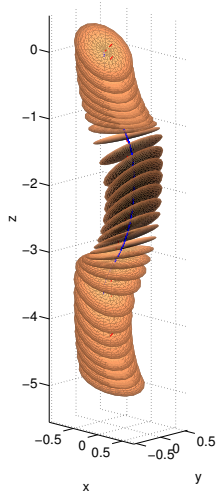

b)

Fig. 1. Vorticity structures and trajectory of a spheroid of aspect ratio $10, m^{*}=0.25$ at $G=115$. Left figures: streamwise vorticity at the same levels: $\omega_{z}= \pm 0.7$. Right figures: kinograms of motion. a) fluid mode, maximum inclination $6^{\circ}$, b) solid mode, maximum inclination $49^{\circ}$.

In figure 2, coexisting solid and fluid modes for spheroids of aspect ratios 3 and 1.1 are illustrated. The kinograms are represented with equal scales of vertical and horizontal axes. The body is on the scale. In figure 2 a) the fluid mode is a slightly oscillating periodic exactly vertical fall unlike that of figure $1 \mathrm{a}$ ). 
The co-existing solid mode yields an oblique trajectory with the spheroid rotating over edge (tumbling regime). This regime is characteristic for rather massive $\left(m^{*} \geq 1\right)$ bodies. To select one of the two modes, appropriate initial condition must be imposed. (E.g., with small and large angular velocity of rotation.)

A similar situation occurs for the almost spherical shape in figure $2 \mathrm{~b}$ ). The trajectory of the fluid mode (represented in the first two figures at left in terms of a kinogram of a short section of motion and a global picture of the path of the body center) is very close to vertical with almost no oscillation of the body. However, if the trajectory of the spheroid center is represented in strongly enlarged horizontal scales, the trajectory appears to be genuinely non-vertical, three-dimensional and chaotic. In contrast, the solid mode presents significant (amplitude of inclination $53^{\circ}$ ), strictly periodic oscillations (the direction of the symmetry axis is visualized as a red dotted line) and the trajectory is planar. Again, the strong solid-fluid interaction enhances the drag by about $10 \%$.
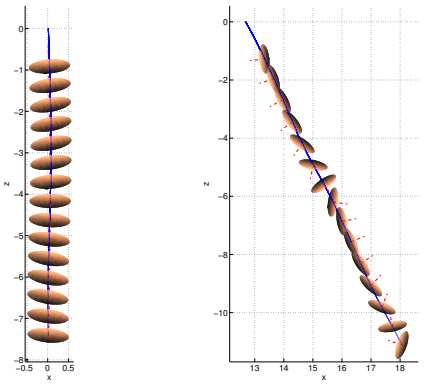

a)
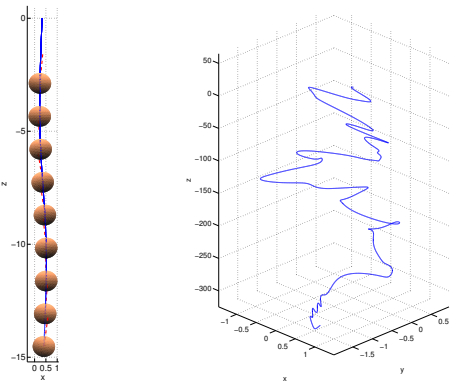

b)

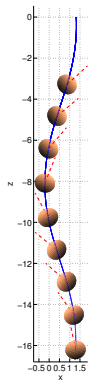

Fig. 2. a) Coexisting fluid mode (left figure) and solid mode (right figure) for spheroid of aspect ratio $3, m^{*}=2$, at $G=125$. b) Coexisting modes for almost spherical spheroid $\chi=1.1, m^{*}=0.5, G=300$. Left two figures: kinogram and trajectory of the center for the fluid mode, right figure: zig-zagging solid mode.

\section{Discussion and conclusion}

Apart from the already mentioned dependence of the trajectories on the initial conditions, the level of external perturbations plays also an important role in the selection of the attractor. The solid modes are, generally, more stable than the fluid ones, which means that the fluid modes can be difficult to observe experimentally if only the motion of the body is registered. This may lead to discrepancies between experimental observations, linear theory and numerical simulations.

Flat spheroids present the three characteristic regimes evidenced experimentally already by Field et al. [9], called "periodic" or "flutter", "intermittent" and 
"tumbling". The numerical study [7] showed that the robust flutter, intermittent and tumbling regimes 'spill over' below the threshold of primary instability of the steady vertical state. The co-existence of solid and fluid unsteady modes illustrated in figure 2 a) characterizes the scenario for large non-dimensionalized masses and that illustrated in figure 1 dominates the region of light spheroids. Experimental observations would tend to overlook the fluid modes while progressive tracking of bifurcations starting from the steady vertical regime might reveal the solid modes only after the fluid ones become unstable. This can yield completely different pictures of the scenario, namely erroneous predictions of the threshold of instability of the vertical trajectories. E.g. observations of a significant upward shift of the threshold of path instability of flat cylinders characterized by a small inertia, were due to the fact that the small oscillations of fluid modes could not be distiguished from experimental noise (see [5]). Similarly, it can be expected, that experiments with more massive bodies will underpredict the instability thesholds.

Almost spherical spheroids $(\chi=1.1)$ present qualitatively the same scenario as spheres [8] overlapped, for light particles $\left(m^{*}<0.25\right.$, i.e. density ratio $\left.<0.5\right)$, with the robust solid zig-zagging mode (flutter) represented in figure $2 \mathrm{~b}$ ). In an experiment, only this regime would be seen for light imperfect spheres. This might be a possible explanation of the surprizing observations reported in ref. $[10]$.

\section{References}

1. Uhlmann M., Doychev, T.: Sedimentation of a dilute suspension of rigid spheres at intermediate Galileo numbers: the effect of clustering upon the particle motion. J. Fluid Mech. 752, 310-348 (2014).

2. Jenny, M., Dušek, J., Bouchet, G.: Instabilities and transition of a sphere falling or ascending freely in a Newtonian fluid. J. Fluid Mech. 508, 201-239 (2004).

3. Ern, P., Risso, F., Fabre, D., Magnaudet, J.: Wake-induced oscillatory paths of bodies freely rising of falling in fluids. Ann. Rev. of Fluid Mech. 44, 97-121 (2011).

4. Chrust, M., Bouchet, G., Dušek, J.: Numerical simulation of the dynamics of freely falling discs. Physics of Fluids 25, 044102 (2013).

5. Chrust, M., Bouchet, G., Dušek, J.:Effect of solid body degrees of freedom on the path instabilities of freely falling or rising flat cylinders. J. Fluids and Structures 47, 55-70 (2014).

6. Tchoufag, J., Fabre, D., Magnaudet, J.: Global linear stability analysis of the wake and path of buoyancy-driven disks and thin cylinders. J. Fluid Mech. 740, 278-311 (2014)

7. Zhou, W., Chrust, M., Dušek, J.: Path instabilities of oblate spheroids, J. Fluid Mech. 883, 445-468 (2017).

8. Zhou, W., Dušek, J.: Chaotic states and order in the chaos of the paths of freely falling and ascending spheres., Int. J. Multiphase Flow 75, 205-223 (2015).

9. Field, S.B., Klaus, M., Moore, M.G.: Chaotic dynamics of falling disks, Nature 388, 252-254 (1997).

10. Horowitz,M., Williamson, C. H. K.: The effect of Reynolds number on the dynamics and wakes of freely rising and falling spheres, J. Fluid Mech. 651, 251-294 (2010). 